\title{
Determinantes da Escolha de Arranjos Institucionais: Evidências na Comercialização de Fertilizantes para Soja ${ }^{1}$
}

\author{
Maristela Franco Paes Leme ${ }^{2}$ \\ Decio Zylbersztajn ${ }^{3}$
}

Resumo: As transações entre os agentes do agronegócio têm deixado de ocorrer estritamente via mercado, passando a serem regidas por diferentes tipos de arranjos institucionais, caracterizados por contratos. No setor de fertilizantes, as empresas intensificaram a oferta de pacotes de insumos aos produtores agrícolas, mais especificamente aos produtores de soja, que são os focalizados neste artigo. Para adquirir fertilizantes, esses produtores podem optar pelo uso de recursos próprios, obtidos em financiamento com terceiros, ou podem ainda recorrer aos referidos pacotes que incluem além dos fertilizantes, soja e recursos financeiros, e que os produtores de soja chamam de "troca”, adquirindo insumos pagando em soja para entrega futura. A motivação do presente trabalho é identificar quais são os fatores determinantes da escolha do produtor de soja entre arranjos alternativos para a aquisição de fertilizantes. Foram realizadas 200 entrevistas com produtores de

1 Os autores agradecem pelas contribuições dos participantes da Oficina PENSA realizado na Faculdade de Economia, Administração da USP, do Congresso da Sociedade Brasileira de Economia e Administração Rural- SOBER e do encontro da Associação Nacional de Pesquisa em Administração- ANPAD. Em especial agradecemos a Sylvia Macchione Saes, Sergio Lazzarini, Cláudio Felisone e Luis. H. M. Villwock pelos comentários em versões anteriores que geraram o presente trabalho.

2 Doutora em Administração - Faculdade de Economia, Administração e Contabilidade (FEA-SP) - Universidade de São Paulo.

3 Professor Titular - Faculdade de Economia, Administração e Contabilidade (FEA-SP) Universidade de São Paulo. 
soja dos estados de Goiás e Mato Grosso. Conclui-se que a formatação de arranjos do tipo bundling pode ser motivada por razões de eficiência, sendo a economia em custos de transação uma dessas razões. A aversão ao risco e a confiança motivam o uso do arranjo troca. Por fim, com relação à escala, os maiores produtores de soja, diferentemente do esperado, utilizaram mais o arranjo troca.

Palavras-chave: Arranjos institucionais, bundling, confiança, custo de transação, fertilizantes, incerteza.

\section{Classificação JEL: D23 e Q13}

Abstract: Transactions among agribusiness agents are not exclusively carried out on the market, being also governed by alternative types of institutional arrangements, some of which determined upon contractual agreement. In the fertilizers sector, the companies intensified the offer of input packages to farmers, more specifically to soybean producers, object of this article. In order to acquire the fertilizers, these producers may choose to use their own financial resources, to obtain resources through financing with third parties, or they may recur to the said packages, which include soybean and financial resources in addition to the fertilizers, referred by the soybean producers to as "exchange", since they acquire fertilizer paying with soybean for future delivery. Thus, the following questions motivate this work: What are the factors that determine the selection of the soybean producer between alternative arrangements for the acquisition of fertilizers? Two hundred interviews were conducted with soybean producers in the States of Goiás and Mato Grosso. To conclude, packages or arrangements as bundling may be stimulated by reasons of efficiency, with transaction costs economy as one of these possible reasons. Risk aversion and trust leads to the use of the exchange arrangement. Finally, in relation to the scale, largest soybean producers use the arrangement exchange in a great deal, differently from what was expected.

Key-words: Institucional arragements, bundling, trust, transation cost, fertilizers, uncertain. 


\section{Introdução}

O relacionamento do setor agrícola com fornecedores de insumos tem passado por alterações no campo organizacional, sendo comum a oferta de soluções em forma de pacotes de insumos e serviços aos produtores agrícolas.

A aquisição de fertilizantes pelos produtores de soja pode ocorrer por meio do mercado spot ou na forma de arranjos alternativos. No primeiro grupo de arranjos, o objeto da transação, tanto para o produtor quanto para o agente comercial, seja misturador, cooperativa ou revendedor, é o fertilizante. Assim, nessa transação típica, no sentido comercializador-produtor segue o fertilizante, e no sentido contrário, o pagamento monetário. Ao realizar o pagamento, o produtor pode utilizar recursos próprios ou com financiamento externo, seja pelos bancos ou outros agentes. Uma das formas do produtor obter recursos para adquirir fertilizante é vender a soja para entrega futura utilizando o chamado contrato de soja verde.

O outro grupo de arranjos é mais complexo: de um lado está o produtor e do outro uma empresa que vende fertilizante e compra soja, sendo que o produtor vende soja para entrega futura para a mesma empresa de quem compra fertilizante, ou seja, o produtor recebe o fertilizante e se compromete a entregar-lhe a soja após a colheita. Essa transação é efetivada por meio de um contrato entre as partes, cujo objeto pode não ser somente o fertilizante, tanto para o produtor de soja quanto para a empresa de fertilizantes. Trata-se, assim, de um pacote composto, além do fertilizante, por soja e por uma operação financeira implícita, o que o caracteriza como bundling ${ }^{4}$. Tal pacote é o que o produtor de soja comumente chama de troca. A Figura 1 mostra os principais tipos de formatação de bundlings que envolvem fertilizantes e soja. O bundling pode ser feito por uma empresa que vende o fertilizante e compra soja (vide caso I), pode ser formatado por empresas que compram soja, mas que, por não lidarem com fertilizantes, adquirem-no de outras empresas para fazer o bundling (vide caso II). Por fim, o bundling pode

\footnotetext{
${ }^{4}$ Bundling refere-se à prática de vender bens em pacotes; no bundling puro, os bens estão disponíveis apenas em pacotes, enquanto no misto, os bens estão disponíveis individualmente ou juntos (ADAMS; YELLEN, 1976; JEULAND, 1984; SCHMALENSEE; WILLIG, 1989).
} 
ser realizado por uma empresa em nome de uma terceira empresa que, além de vender fertilizante, também adquire soja (vide caso III).

Figura 1 - Principais tipos de formatação do bundling de fertilizantes e soja

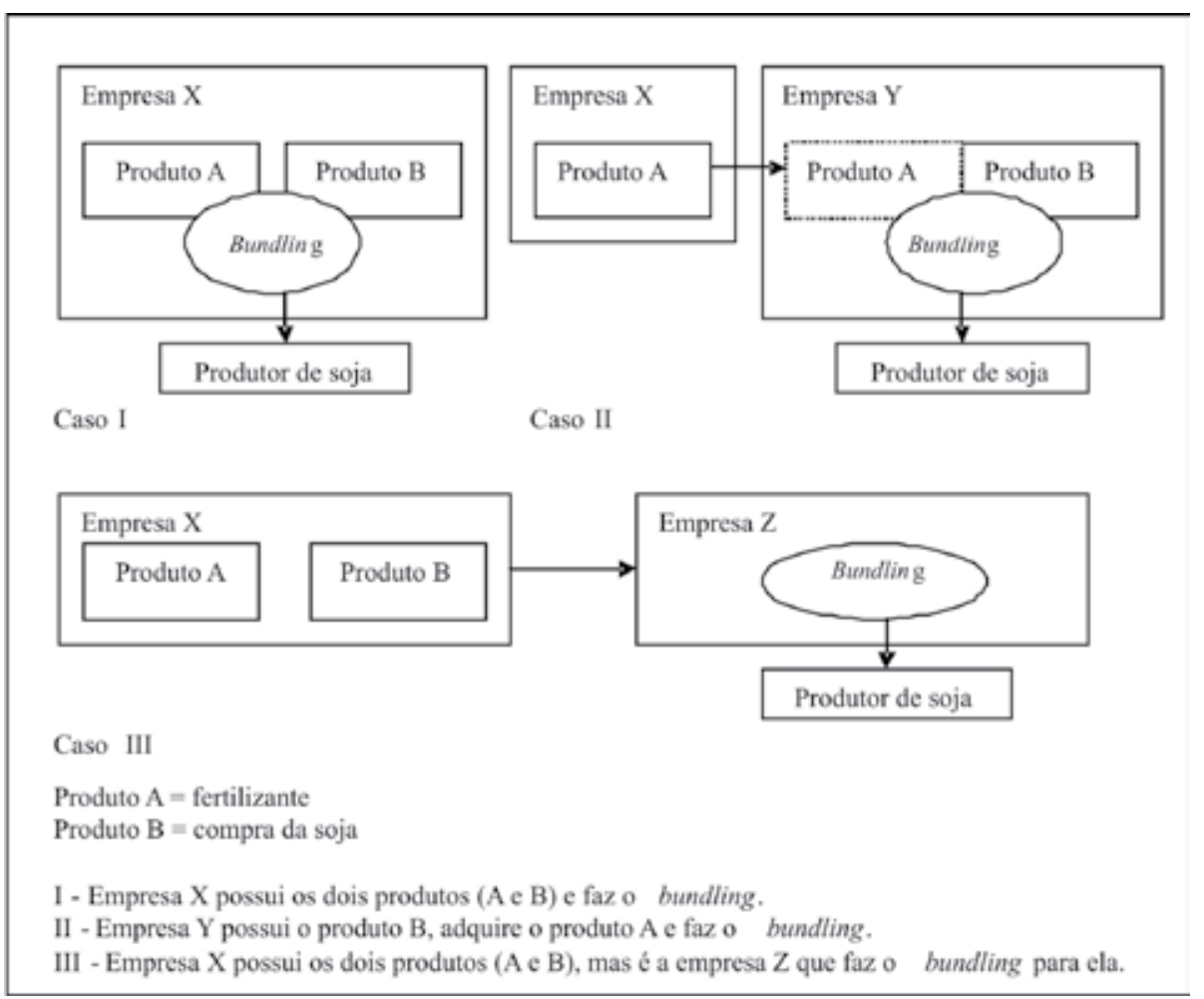

Fonte: os autores.

As misturadoras de fertilizantes são também as originadoras de grãos. A operação de troca mesmo para essas empresas é complexa: elas antecipam recursos para o produtor adquirir fertilizantes e em troca recebem a soja após a colheita. Para essas empresas o objeto da transação não é o fertilizante em si, que não pode ser separado da soja e do próprio crédito. Muitas vezes a antecipação dos recursos é viabilizada por meio de operações financeiras realizadas pelas empresas que, por isso, cobram um spread pela antecipação do crédito para os produtores. Na maior parte das vezes existem bancos envolvidos na 
transação oferecendo crédito, tornando a gestão do risco um fator relevante na operação. Para o agricultor o objeto da transação nesse tipo de arranjo é múltiplo, ao mesmo tempo negocia-se a compra de fertilizante e a venda da soja.

Dentre os arranjos que envolvem fertilizantes e soja, existem diferenciações dependendo da empresa com relação à fixação do preço da soja, pois se pode utilizar a modalidade de preço fixo ou a fixar. Além disso, pode haver variações na data de pagamento pela soja.

O objetivo deste artigo é estudar empiricamente os fatores determinantes da escolha do produtor de soja entre arranjos alternativos para aquisição de fertilizante. Mais especificamente, focaliza os arranjos em que o produtor compra fertilizante e vende soja com entrega futura para a mesma empresa. Acredita-se que a percepção dos custos de transação, da incerteza e da confiança por parte do produtor pode influenciar a sua escolha entre os arranjos institucionais. O artigo contribui para a literatura sobre formas complexas cuja interpretação gravita entre o poder de monopólio ou arranjos eficientes.

Este artigo está estruturado em cinco partes. Inicialmente, discute os arranjos alternativos que podem ser utilizados para aquisição de fertilizantes pelos produtores de soja e introduz as possíveis variáveis associadas com a escolha entre os referidos arranjos. Em seguida, apresenta o referencial teórico utilizado para suportar as variáveis mencionadas. Depois discute o método de pesquisa que inclui os modelos empíricos e as variáveis. Os dados obtidos em entrevistas com 200 produtores de soja de Goiás e Mato Grosso são analisados em seguida. Por fim, o artigo apresenta conclusões, as limitações e sugestões para futuras pesquisas.

\section{Quadro Conceitual}

É importante conhecer as variáveis que podem influenciar a escolha dos produtores de soja entre arranjos alternativos no momento da aquisição de fertilizantes. O custo de transação, inicialmente discutido por Coase (1937), é uma das explicações plausíveis, e compreende tanto os custos ex-ante, relacionados ao desenho, negociação e montagem de salvaguardas dos contratos, bem como os custos ex-post, associados ao monitoramento, renegociação e custos incorridos com o ordenamento 
privado e com a justiça, no caso de quebras contratuais. Neste artigo, consideram-se principalmente os custos de transação ex-ante, ou seja, os custos envolvidos da negociação até a assinatura do contrato entre as partes. Assim, chamou-se esta variável de custo de negociação (CN) para captar a percepção dos produtores de soja do custo de negociar, analisar e formatar contratos, tendo em vista que se acredita que esse custo influencia a decisão de utilizar cada um dos arranjos alternativos disponíveis para aquisição de fertilizantes. Acredita-se que os contratos sejam utilizados em vez do mercado, para economizar em custos de transação, embora a especificidade dos ativos não seja a variável chave, conforme o modelo proposto por Williamson (1985), onde a forma organizacional é a variável endógena, e a especificidade dos ativos, a variável explicativa.

Segundo Pennings e Leuthold (2000), uma das limitações de seu estudo foi não considerar os custos de transação entre as variáveis explicativas do uso de contratos futuros pelos produtores rurais. Neste artigo, essa variável não é considerada diretamente, mas o custo de negociação constitui parte do chamado custo de transação.

A incerteza é outro construto utilizado para explicar a escolha entre arranjos institucionais alternativos. Williamson (1996) afirma que elevada incerteza aumenta a probabilidade das estruturas de governança polares (mercado ou hierarquia). Embora a eficácia de todas as formas de governança possa deteriorar-se diante de distúrbios mais freqüentes, a forma híbrida é a mais suscetível, pois a adaptação nesta última requer consentimento mútuo (WILLIAMSON, 1991).

Não há consenso na literatura, com relação à divisão da incerteza em duas partes, sendo uma mensurável e a outra não, onde a primeira é definida como risco. Para Knight (1921) existe uma diferença fundamental entre a incerteza determinada ou risco e a indeterminada ou não-mensurável. De acordo com Rocha (2002), a teoria da escolha sob incerteza ou teoria da utilidade esperada de Von Neumann-Morgenstern, desconsiderou a diferença entre as incertezas mensurável e não mensurável proposta por Knight (1921). Neste artigo, para representar a incerteza, utiliza-se uma medida de risco, independente de este não captar toda a dimensão da incerteza.

Assim, como em Pennings e Leuthold (2000), dividiu-se o risco em 
duas variáveis. A primeira é a atitude com relação ao risco (AR) que pode ter papel importante na escolha do arranjo institucional utilizado. $\mathrm{O}$ risco precisa ser primeiro percebido, para depois responder-se a ele. Assim, a segunda variável é a exposição percebida ao risco (EPR) que pode ser definida como a avaliação do produtor de soja do risco inerente em uma situação.

O terceiro construto considerado é a confiança (CONF). Esta tem sido considerada em vários trabalhos que tratam as relações verticais (ANDERSON e NARUS, 1990; GANESAN, 1994; CLARO, 2003) e horizontais (SMITH e BARCLAY, 1997) entre agentes. Para definir e delimitar a noção de confiança, Williamson (1996) emprega a razão econômica calculativa da qual a economia dos custos de transação é variante.

Neste artigo, considera-se a dimensão inter-organizacional da confiança, ou seja, a confiança com relação à empresa. Como Nooteboom et al. (1997), trata-se a confiança como uma percepção do indivíduo com respeito à organização envolvida no processo de troca. Claro (2003) considerou diferentemente tanto a dimensão inter-organizacional da confiança quanto a dimensão inter-pessoal. A confiança não é uma condição objetiva e varia mesmo entre indivíduos que estão em condições idênticas. Tal como Malhotra e Murnighan (2002), considera-se que a confiança depende em grande parte da interação histórica das partes. Assim, interações repetidas provêem um significado central para construir ou aumentar a confiança.

Por fim, o último construto é a escala (ESC). De acordo com Spiller e Zelner (1997), a alta sofisticação do consumidor desfavorece as estratégias de bundling 5 . Neste artigo, assume-se que a sofisticação do produtor de soja esteja relacionada à escala de produção, tendo em vista que os maiores produtores possuem nível tecnológico mais elevado e adotam instrumentos mais modernos de comercialização da safra, como contratos futuros e de opções.

\footnotetext{
${ }^{5}$ Quanto à motivação para uso do bundling, para Adams e Yellen (1976) alguns atribuem-no à economia de custo na produção, transação e informação associada com a venda do pacote. Segundo Nalebuff (1999), uma explicação óbvia para muitos bundles é que a empresa pode integrar os produtos melhor do que seus clientes. Para Schmalensee (1984, apud Schmalensee e Willig, 1989), o verdadeiro papel do bundling é reduzir a heterogeneidade no que se refere à avaliação dos consumidores. Chen (1997) mostrou o potencial do bundling como um facilitador, ou seja, uma forma para duas empresas competidoras se diferenciarem.
} 
Determinantes da Escolha De Arranjos Institucionais: Evidências Na Comercialização De Fertilizantes Para Soja

\section{Metodologia}

Tomando por base a economia dos custos de transação, define-se para o presente estudo que a transação objeto de análise é aquela entre produtores de soja e empresas de fertilizantes, focalizando o fertilizante fornecido para a produção de soja nos estados de Goiás e de Mato Grosso. As entrevistas foram realizadas de forma individual com os produtores de soja, em maio de 2004, por telefone, seguindo o questionário cujas principais perguntas foram feitas utilizando escalas. Seguindo a metodologia da escala tipo Likert, os entrevistados foram solicitados a informar seu grau de concordância /discordância com relação às afirmações. Para cada célula de resposta foi atribuído um número de 1 a 5 que reflete a direção da atitude do entrevistado para cada afirmação. Quando a informação era favorável, atribuiu-se o número 5, caso houvesse concordância total, e 1 caso houvesse discordância total. Para afirmação desfavorável, atribuiu-se 1 em caso de concordância total e 5 em caso de discordância total.

Como cada produtor de soja entrevistado poderia ter mais de uma propriedade em Goiás e no Mato Grosso, colocou-se no questionário uma instrução para que o entrevistador, ao perguntar sobre a localização da propriedade, focalizasse a sua propriedade principal. Nas questões relacionadas à área plantada e ao tipo de arranjo utilizado para aquisição de fertilizante, por decorrência, também foi focalizada apenas a propriedade principal, o que evita dúvidas no momento da resposta e problemas oriundos do fato do produtor adquirir fertilizantes com variados tipos de arranjos em propriedades diferentes.

Os produtores de soja entrevistados foram selecionados aleatoriamente em um banco de dados pertencente a uma empresa de consultoria que dentre outras atividades faz levantamentos diversos entre esses produtores e vende serviços de informação. Esse banco de dados é privado e possui um total de 1.000 produtores de soja nos estados de Goiás e Mato Grosso. Foram entrevistados produtores que utilizaram diferentes tipos de arranjos para aquisição de fertilizantes, sendo que cada produtor cadastrado no banco de dados teve igual probabilidade de ser selecionado.

Foram feitas 200 entrevistas com produtores de soja, sendo 68 em 
Goiás e 132 no Mato Grosso, o que representa 1,95\% do total de produtores de soja em Goiás e 4,80\% dos produtores de soja no Mato Grosso, se considerarmos o Censo Agropecuário de 1996. A não aleatoriedade da amostra pode implicar no distanciamento dos pressupostos de normalidade gerando potencial viés nos resultados e na robustez dos testes estatísticos. Por outro lado o número de entrevistas não caracteriza estritamente a metodologia como sendo de múltiplos estudos de caso.

\subsection{Variáveis e hipóteses}

O presente estudo propõe que um conjunto de fatores definidos como custo de negociação $(\mathrm{CN})$, exposição percebida ao risco (EPR), atitude ao risco (AR), confiança (CONF) e escala (ESC) explique a escolha do arranjo compra de fertilizantes por meio da venda da soja para entrega futura para a mesma empresa (ARR).

Quando se utiliza o pacote fertilizante/soja, em um mesmo momento são transacionados vários itens, ou seja, o produtor de soja em vez de despender tempo com a busca de financiamento, compra do fertilizante antes do plantio e venda da soja após a colheita, negocia estes itens conjuntamente, otimizando o tempo de negociação. A primeira hipótese definida no estudo é definida a seguir:

H1: A percepção de economia na negociação motiva o produtor a optar pela utilização do pacote fertilizante/soja, ou seja, a comprar fertilizantes e vender soja para uma mesma empresa em vez de fazê-lo com empresas diferentes.

Ao utilizar o pacote em questão, o produtor, no momento da aquisição do fertilizante, conhece a relação de troca fertilizante/soja, ou seja, independentemente do preço da soja após a colheita, o produtor sabe quanto do seu produto será empregado para quitar o fertilizante. Com o conhecimento da relação de troca e travamento antes do plantio, do preço da soja, a incerteza do produtor é reduzida. Assim, a segunda hipótese é definida como:

H2: A exposição percebida e a aversão ao risco motivam o produtor a optar pela utilização do pacote fertilizante/soja.

Ao escolher a utilização do pacote, o produtor aceita a relação de preço fertilizante/ soja das empresas de fertilizantes, apesar de acreditar 
que estas podem deter mais informações sobre o comportamento de preços futuros da soja. No entanto, a confiança depositada nas empresas de fertilizantes determina que o produtor aceite a relação de troca ofertada, principalmente porque existe um histórico de transações, e de reputação e pela expectativa de que a transação possa repetir-se em anos subseqüentes. Dessa forma, a terceira hipótese é:

H3: A confiança depositada pelo produtor de soja na empresa de fertilizante motiva-o a optar pela utilização do pacote fertilizante/soja.

Segundo Spiller e Zelner (1997), a alta sofisticação do consumidor desfavorece as estratégias de bundling. No caso da soja, a alta sofisticação está relacionada, principalmente, à escala de produção, pois quanto maior a escala, mais alto o nível de tecnologia empregado para plantio, manejo e colheita da safra. Além disso, os grandes produtores de soja utilizam mecanismos de gerenciamento de risco diferenciados, como o mercado futuro e de opções, operando muitas vezes diretamente na Bolsa de Chicago. Assim, a quarta e última hipótese é:

H4: Quanto maior a escala do produtor de soja, menor é a utilização do pacote fertilizante/soja.

\subsection{Medidas e método de análise dos dados}

As variáveis; custo de negociação (CN), atitude ao risco (AR), exposição percebida ao risco (EPR) e confiança (CONF) não podem ser medidas diretamente, ou seja, não são observadas, sendo chamadas de variáveis latentes. Computa-se esse tipo de variável por meio de indicadores que são as variáveis observadas. Utilizaram-se três indicadores para cada uma das variáveis latentes, com exceção da variável exposição percebida ao risco, para a qual foram utilizados quatro indicadores, conforme mostra a Tabela 1. Tal metodologia foi utilizada por Pennings e Leuthold (2000) e por Claro (2003). Conforme mostra o questionário, cada indicador é uma afirmação para a qual o entrevistado teve que atribuir valor.

A variável escala (ESC) é medida pela área plantada de soja na última safra na principal propriedade; neste sentido, a escala é a única variável independente métrica ou não escalar. 
Tabela 1 - Resumo das variáveis latentes e seus indicadores

\begin{tabular}{lc}
\hline Variáveis Latentes & Indicadores \\
\hline $\mathrm{CN}$ & CN0, CN1, CN2 \\
EPR & EPR0, EPR1, EPR2, EPR3 \\
\hline AR & AR0,AR1,AR2 \\
\hline CONF & CONF0,CONF1,CONF2 \\
\hline
\end{tabular}

Fonte: os autores

Como as variáveis utilizadas, com exceção da variável escala (ESC), são medidas por um grupo de variáveis observadas ou indicadores, fez-se análise fatorial confirmatória para verificar as suas propriedades com relação a sua validade e confiabilidade. Além disso, analisou-se também o alfa de Cronbach para examinar a confiabilidade. A validade refere-se à extensão na qual um conjunto de medidas representa um conceito, ou seja, a variável latente. A confiabilidade refere-se à consistência dos indicadores com relação à medição da variável latente. Nunnally e Bernstein (1994) apresentam os métodos desenvolvidos para testar a validade e a confiabilidade das variáveis.

O alfa de Cronbach é uma medida de confiabilidade comumente usada, seu valor fica entre zero e um, sendo que valores maiores indicam maior confiabilidade dos indicadores para capturar o conceito da variável latente. Segundo Hair et al. (1998), a prática comum é aceitar valores de alfa superiores a 0,70 , sendo aceitáveis valores superiores a 0,60 .

O modelo de análise fatorial confirmatória assume que as variáveis observadas (indicadores) são geradas por um menor número de variáveis não observadas (variáveis latentes). A relação das variáveis observadas e não observadas pode ser representada pela equação 1:

$$
\text { (1) } X=\Lambda \mathrm{k}+\zeta
$$

onde $\mathrm{X}$ é o vetor q $\mathrm{x} 1$ dos $\mathrm{n}$ grupos de variáveis observadas, $\mathrm{k}$ é o vetor $\mathrm{n} \times 1$ dos fatores, $\Lambda$ é a matriz q x $\mathrm{n}$ dos coeficientes da regressão relacionando as variáveis observadas aos fatores e $\zeta$ é o vetor q x 1 do termo de erro das variáveis observadas. 
A análise fatorial confirmatória estima as relações entre as variáveis observadas e as variáveis latentes, sendo que várias variáveis latentes podem ser estimadas simultaneamente. Conforme sugerido por Hair et al. (1998), utiliza-se a matriz de correlação como matriz de entrada de dados. A matriz de correlação é preferível à de covariância, pois torna possível a comparação direta dos coeficientes dentro de um modelo. Com relação ao processo de estimação, utilizou-se a técnica ADF, Asymptotically Distribution Free, que segundo Hair et al. (1998) e Sharma et al. (1989) é a mais utilizada para casos de dados não normais. Baseado na prática comum, segundo Hair et al. (1998), de que os valores de skewness e kurtosis não devem exceder /1/, a maior parte das variáveis observadas, neste artigo, não são normalmente distribuídas; assim, o método de máxima verossimilhança não é recomendável ${ }^{6}$.

Em termos de valores de referência para análise dos resultados da análise fatorial confirmatória, recomenda-se que o resíduo padronizado seja inferior ao $/ 2,58 /$, que o $\lambda$ padronizado de cada variável observada seja maior que 0,60 com o valor do t maior que 2,0 .

Em termos de medidas de ajustamento para verificar até que ponto o modelo é bem especificado para os dados, Hair et al. (1998) recomendam que sejam analisadas as medidas absolutas (estatística qui-quadrado, GFI - goodness of fit index, RMSR - root mean square residual), as medidas incrementais como NFI (normed fit index) e as medidas parcimoniosas ajustadas pelos graus de liberdade como AGFI - adjusted GFI for degree of freedom. Em termos de valores de referência, recomenda-se que as medidas GFI, AGFI, NFI sejam maiores ou iguais a 0,90 e a medida RMSR próxima de zero. A Tabela 2 mostra um resumo dos valores de referência de cada medida. As medidas GFI e AGFI avaliam quão melhor o modelo de medida proposto ajusta-se aos dados comparativamente com qualquer outro modelo. O RMSR mede a média da variância do resíduo que não pode ser explicada pelo modelo. O NFI é uma medida incremental para a estatística qui-quadrado e é utilizada para comparar o ajustamento do modelo estimado com o ajustamento de um modelo nulo, no qual se assume que todas as variáveis

${ }^{6}$ No caso de dados não normais, o uso do método da máxima verossimilhança implica erros padrão instáveis, embora os parâmetros estimados não sejam viesados (SHARMA et al.,1989). 
são não correlacionadas. Se o ajustamento do modelo nulo é bom, o ajustamento do modelo estimado também será.

Tabela 2 - Resumo dos valores de referência das medidas de ajustamento

\begin{tabular}{lc}
\hline Medidas & Valores de referência \\
\hline GFI, AGFI, NFI & $>0.90$ \\
RMSR & Próximo a 0 \\
\hline
\end{tabular}

Fonte: Hair et al. (1998)

Hair et al. (1998) sugerem que, após o exame das medidas de ajustamento para o modelo, sejam feitas as análises das medidas de cada construto. Embora o alfa de Cronbach seja utilizado para verificar a confiabilidade, outras medidas importantes, segundo Hair et al. (1998), para avaliar a confiabilidade são a confiabilidade composta e variância extraída que são calculadas a partir dos resultados da análise fatorial confirmatória. A confiabilidade composta é uma medida da consistência interna dos indicadores de uma variável latente e representa o grau em que os indicadores estão relacionados com essa variável. De acordo com Hair et al. (1998), os guias sugerem que o valor da confiabilidade composta seja maior que 0,70 , mas valores inferiores também podem ser aceitáveis em pesquisas exploratórias.

A variância extraída por sua vez reflete toda a variância dos indicadores sob a variável latente, sendo que os valores de referência sugeridos devem exceder 0,50 para cada variável latente.

Com relação à multicolinearidade, valores na matriz de correlação excedendo 0,9 devem ser examinados, corrigidos e, se necessário, eliminada a variável latente.

A análise fatorial confirmatória estima as variáveis não observadas a partir das variáveis observadas, mas não provêinformação sobre a relação entre as variáveis independentes e a dependente. Para testar as relações entre as variáveis latentes, que no caso são as variáveis independentes, e a variável dependente, utilizou-se o modelo de equações estruturais (Structural Equation Modeling - SEM), pois as variáveis não observadas estão sujeitas a erros de medida, podendo resultar em coeficientes viesados quando estimados por regressão linear. Este modelo estima 
- Determinantes da Escolha De Arranjos Institucionais: Evidências Na Comercialização De Fertilizantes Para Soja

simultaneamente as variáveis independentes (não observadas) a partir das variáveis observadas e a relação entre estas variáveis e a variável dependente. Assim, segundo Hair et al. (1998), o modelo de equações estruturais é atrativo, pois provê um método que lida simultaneamente com múltiplas relações com eficiência estatística e possui a habilidade de aferir a relação de forma compreensível, oferecendo uma transição da análise fatorial exploratória para a análise confirmatória. Com o modelo de equações estruturais estima-se simultaneamente uma série de equações de regressão múltipla separadas, mas interdependentes, por meio da especificação de um modelo estrutural utilizado pelo programa estatístico, que neste caso é o SAS. A característica principal de tal modelo é a capacidade de incorporar variáveis latentes na análise.

O presente estudo propõe que o custo de negociação $(\mathrm{CN})$, a exposição percebida ao risco (EPR), a atitude ao risco (AR), a confiança (CONF) e a escala (ESC) explicam a escolha do arranjo compra de fertilizantes por meio da venda da soja para entrega futura para a mesma empresa (ARR). Os outros arranjos utilizados pelos produtores de soja para aquisição de fertilizantes, que não o relacionado com a venda da soja para entrega futura para a mesma empresa, foram agrupados em outra categoria. Assim, como o somatório do uso do arranjo compra de fertilizantes por meio da venda da soja para entrega futura para a mesma empresa com o uso dos outros arranjos totaliza $100 \%$, somente o percentual de uso do arranjo compra de fertilizantes por meio da venda da soja para entrega futura para a mesma empresa foi utilizado como variável dependente no modelo de equações estruturais.

\section{Resultados}

Dos produtores de soja entrevistados, conforme mostra a Tabela 3, 34\% possuem propriedade principal no estado de Goiás e $66 \%$ no estado de Mato Grosso, o que representa 1,95\% do total de produtores em Goiás e 4,80\% dos produtores no Mato Grosso, considerando o Censo Agropecuário de 1996.

Dos produtores entrevistados, 16,5\% afirmaram plantar soja há menos de cinco anos, enquanto $21 \%$ mencionaram plantar soja há mais de 20 anos. Assim, conforme a Tabela 4, os produtores da amostra estão 
distribuídos com relação ao tempo de plantio de soja, o que reflete que foram entrevistados produtores com experiências diferenciadas.

Tabela 3 - Produtores de soja da amostra por estado (\%)

\begin{tabular}{lc}
\hline Estado & Produtores de soja \\
\hline Goiás & 34 \\
Mato Grosso & 66 \\
\hline
\end{tabular}

Fonte: os autores

Tabela 4 - Distribuição do período de tempo em que os produtores da amostra plantam soja

\begin{tabular}{|c|c|}
\hline Período de tempo (anos) & Produtores (\%) \\
\hline $1-4$ & 16,5 \\
\hline $5-8$ & 12,5 \\
\hline $9-12$ & 11,5 \\
\hline $13-16$ & 11,0 \\
\hline $17-20$ & 27,5 \\
\hline$>20$ & 21,0 \\
\hline
\end{tabular}

Fonte: os autores

Tabela 5 - Distribuição da escala dos produtores de soja da amostra segundo a área plantada

\begin{tabular}{lc}
\hline Escala (ha) & Produtores (\%) \\
\hline$<1.000$ & 45,0 \\
$1.001-2.000$ & 20,5 \\
$2.001-3.000$ & 8,5 \\
$3.001-4.000$ & 8,0 \\
$4.001-5.000$ & 4,5 \\
$>5.000$ & 13,5 \\
\hline
\end{tabular}

Fonte: os autores

Com relação ao tamanho dos produtores de soja da amostra, $45 \%$ deles plantam menos de 1.000 hectares, 20,5\% plantam entre $1.001 \mathrm{e}$ 2.000 hectares, $21 \%$ entre 2.001 e 5.000 e $13,5 \%$ plantam mais de 5.000 hectares, conforme a Tabela 5 . 
Por fim, no que diz respeito ao tipo de arranjo utilizado para aquisição de fertilizantes, a Tabela 6 informa que 31,5\% dos produtores entrevistados compraram fertilizantes, total ou parcialmente, por meio da venda de soja para entrega futura para a mesma empresa, arranjo comumente denominado troca.

Os recursos próprios foram utilizados para o plantio de soja por $24,5 \%$ dos produtores, o que reflete de certa forma sua capitalização em virtude dos bons resultados alcançados em anos subseqüentes com a cultura. A correlação entre uso de recursos próprios e a escala dos produtores de soja é de 0,09. Apesar de baixa, tal correlação é positiva, o que indica que os maiores produtores utilizam os recursos próprios em maiores proporções. A capitalização dos grandes produtores de soja, comparativamente aos menores, é uma possível explicação para a correlação positiva.

Com relação ao plantio com o uso de financiamento oficial ou de bancos e empresas diversas, $25 \%$ dos produtores adotaram essa modalidade. Cabe destacar que no caso do financiamento oficial para plantio de soja, apenas $\mathrm{R} \$ 200$ mil foram disponibilizados por CPF na safra $2003 / 2004$ a juros de $8,75 \%$ ao ano. No Centro Oeste, o financiamento para aquisição de fertilizantes não formulados também proveio de recursos do FCO, à taxa de juros que variou entre 8,75 a 10,75\% ao ano, dependendo da classificação do produtor quanto à sua receita bruta anual. A Tabela 6 demonstra que, mesmo em se tratando somente de fertilizantes, os produtores de soja utilizam diferentes arranjos para adquiri-los em um único ano/safra.

Ao fazer a análise fatorial confirmatória as variáveis latentes custo de negociação $(\mathrm{CN})$, exposição percebida ao risco (EPR), atitude ao risco (AR) e confiança (CONF)) foram combinadas duas a duas, devido ao fato de três das variáveis latentes possuírem apenas três variáveis observadas, o que faz com que o grau de liberdade do modelo estimado seja zero. Assim, as medidas de ajustamento do modelo (GFI, AGFI, RMSR e NFI) não podem ser calculadas. Segundo Hair et al. (1998), essa metodologia é prática comum na literatura. Ao serem combinadas as variáveis duas a duas, as medidas de ajustamento representam o modelo que contempla ambas as variáveis, mas os coeficientes $(\lambda)$ são particulares de cada variável. Assim, a análise fatorial confirmatória de 
$\mathrm{CN}$ foi realizada com EPR, a de AR com $\mathrm{CN}$ e a de CONF foi realizada com CN, ou seja, $\mathrm{CN}$ entrou em todos os modelos. Embora EPR tivesse quatro variáveis observadas, a análise fatorial confirmatória de EPR também teve de ser realizada de forma conjunta, pois EPR3 apresentou problema de singularidade da matriz e teve de ser eliminada.

Tabela 6 - Distribuição dos produtores de soja segundo o tipo de arranjo utilizado para aquisição de fertilizantes

\begin{tabular}{lc}
\hline Tipo de arranjo & Produtores (\%) \\
\hline Recursos próprios & 24,5 \\
Financiamento & 25,0 \\
Troca & 11,5 \\
Recursos próprios / financiamento & 19,0 \\
Recursos próprios / troca & 6,5 \\
Financiamento / troca & 10,5 \\
Recursos próprios / financiamento / troca & 3,0 \\
\hline
\end{tabular}

Fonte: os autores

Antes de realizar a análise fatorial confirmatória, os dados da amostra foram analisados para verificar a normalidade univariada e multivariada. A opção por utilizar a técnica ADF para realizar as estimações, conforme foi discutido, decorreu do fato de a maior parte das variáveis ter apresentado valores de skewness e/ou kurtosis maiores que o $/ 1 /$.

Os dados foram examinados para verificar a presença de outliers, inclusive, a princípio, a partir da análise gráfica da normalidade dos dados, foram eliminados seis produtores de soja que produziam mais de 20.000 hectares. No entanto, os resultados encontrados não representaram melhoria nos testes de normalidade. Assim, os resultados apresentados neste artigo são da amostra completa, ou seja, de 200 produtores de soja.

A variável latente, custo de negociação $(\mathrm{CN})$, foi medida por três variáveis observadas, ou seja, utilizaram-se três afirmações para as quais o entrevistado teve de atribuir valores de 1 a 5 . A variável latente exposição percebida ao risco (EPR) foi medida por quatro variáveis observadas. Três variáveis observadas foram utilizadas para medir o 
construto atitude ao risco (AR) e, por fim, a variável latente confiança (CONF) foi medida por três variáveis observadas.

As medidas GFI, AGFI, RMSR e NFI ficaram dentro do valor recomendado para todas as variáveis latentes.

CNO foi o único indicador de $\mathrm{CN}$ cujo valor do $\mathrm{t}$ dos coeficientes $(\lambda)$ ficou abaixo do limite recomendável, embora o valor do t também não tenha sido significativo. $O$ valor do t do coeficiente $(\lambda)$ de EPR2 ficou abaixo do valor esperado, que é $/ 2 /$, ou seja, não foi significativo. Apenas o valor do coeficiente de EPR0 foi maior de 0,60 e significativo. Todos os demais coeficientes $(\lambda)$ são superiores a 0,60 e os erros padrão são inferiores a /2,58/.

Analisando as medidas calculadas com base nos resultados da análise fatorial confirmatória, observa-se que tanto a confiabilidade composta quanto a variância extraída de todas as variáveis latentes são maiores que o limite mínimo recomendado por Hair et al. (1998). Os alfas de Cronbach para as variáveis CN, EPR, AR, CONF ficaram dentro de um limite aceitável, indicando, juntamente com os resultados da análise fatorial confirmatória, a validade e a confiabilidade da variável em análise.

Para analisar as relações entre as variáveis independentes e a variável dependente, utilizou-se o modelo de equações estruturais. Verificou-se a validade e a confiabilidade das variáveis latentes formadas a partir de uma série de variáveis observadas. Cada uma das variáveis latentes analisadas foi levantada com base no referencial teórico. O modelo estrutural relaciona as variáveis independentes e a dependente, enquanto o modelo de medidas especifica as variáveis observadas de cada variável latente.

A Tabela 7 mostra os resultados do modelo de equações estruturais estimado. Ao estimar tal modelo, verificou-se que as variáveis observadas CN0 e EPR3 apresentaram multicolinearidade, razão pela qual foram eliminadas.

A Tabela 7 apresenta as medidas de ajustamento (GFI, AGFI, RMSR e NFI) do modelo de equações estruturais estimado que podem ser aceitas conforme os critérios apresentados na Tabela 2. Com relação à significância dos coeficientes do modelo, apenas o valor do t da variável exposição percebida ao risco (EPR) foi inferior a /2/, portanto, não 
significativo. Todos os erros padrão ficaram dentro do limite esperado, que é $/ 2,58 /$.

Tabela 7 - Resultado do modelo de equações estimado

\begin{tabular}{lccc}
\hline Indicadores & Coeficiente & t do coeficiente & erro padrão \\
\hline CN & 0,27 & 6,06 & 0,04 \\
EPR & 0,001 & 0,02 & 0,05 \\
AR & 0,12 & 2,79 & 0,04 \\
CONF & 0,14 & 2,88 & 0,04 \\
ESC & 0,94 & 51,32 & 0,01 \\
\hline \multicolumn{4}{l}{ GFI $=0,99 ;$ AGFI $=0,9972 ;$ RMSR $=0,14 ;$ NFI $=0,99$} \\
\hline
\end{tabular}

Fonte: os autores

Conforme esperado, o custo de negociação $(\mathrm{CN})$ tem relação positiva com a escolha do arranjo aquisição de fertilizantes por meio da venda da soja para entrega futura para a mesma empresa, ou seja, os produtores com maior nível de concordância com relação à otimização de tempo, economia de recursos e rapidez do processo vinculado ao referido arranjo são os produtores que o utilizaram em maior percentual.

Os coeficientes da exposição percebida ao risco (EPR) e da atitude ao risco (AR) apresentaram sinal positivo de acordo com o esperado, embora o valor do t da variável exposição percebida ao risco não tenha sido significativo.

Com a aquisição de fertilizantes por meio da venda de soja para entrega futura para a mesma empresa, o produtor de soja fica ciente da relação de troca insumo/ produto no que se refere pelo menos ao fertilizante, ou seja, independentemente do preço da soja após a colheita, sabe-se quanto do seu produto será empregado para pagar os fertilizantes. Assim, o produtor fica protegido no que se refere a esse item. Com o conhecimento da relação de troca e travamento do preço da soja antes do plantio, a incerteza do produtor de certa forma é reduzida. De acordo com o discutido, utilizam-se duas variáveis relacionadas ao risco para captar o conceito de incerteza, independentemente de o risco não esgotar toda a dimensão desta última. Essas variáveis são a exposição percebida ao risco e a atitude ao risco.

Com relação à variável exposição percebida ao risco, embora esta 
não tenha sido significativa para explicar o uso do arranjo troca, verifica-se que existe uma relação positiva entre a percepção de exposição ao risco e o percentual de uso do arranjo em questão, ou seja, a percepção de alta exposição ao risco aumenta o uso do arranjo troca pelo produtor de soja. Não foram encontrados indícios teoricamente aceitáveis para supor que a amostra não fosse homogênea para justificar a sua segmentação. Com respeito à atitude ao risco, verifica-se que quanto mais averso ao risco, mais o produtor de soja utiliza o arranjo troca.

A variável que captura confiança apresentou sinal de acordo com o esperado, ou seja, quanto maior a confiança, maior o percentual de uso do arranjo troca. O fato de o produtor de soja ter afirmado que sempre compra fertilizantes da mesma empresa e que ele confia naquela empresa tem relação positiva com o uso do referido arranjo. Trata-se a confiança como uma percepção do indivíduo com respeito à organização envolvida no processo de troca, ou seja, considera-se a dimensão inter-organizacional da confiança, que se aproxima do conceito de reputação. Tal como Malhotra e Murnighan (2002), considera-se também que a confiança depende em grande parte da interação histórica das partes. Ao escolher a utilização do arranjo troca, o produtor aceita a relação de preço fertilizante/soja da empresa de fertilizante, consciente de que as empresas podem deter mais informações sobre o comportamento futuro dos preços, em parte pela confiança nelas depositada. Além disso, existe um histórico de transações, reputação e repetição do uso do arranjo em mais de uma safra. Em contato com o dirigente de uma grande empresa de fertilizante, constatou-se que existe alta incidência de produtores que utilizam o arranjo troca em anos subseqüentes.

Por fim, a variável "escala”, diferentemente do esperado, foi positivamente relacionada ao uso do referido arranjo, ou seja, os maiores produtores de soja utilizaram mais o arranjo troca. A priori, esperava-se que os maiores produtores utilizassem menos o arranjo troca, pois eles mesmos seriam capazes de formatar seus próprios arranjos. Pode ser que a utilização da variável escala como proxy para a variável sofisticação empregada por Spiller e Zelner (1997), não tenha sido adequada, ou seja, os maiores produtores de soja não são os mais sofisticados. Nesse caso, não se poderia contestar a afirmação de tais autores de que quanto maior a sofisticação, menor a utilização de arranjos do tipo 
bundling. $\mathrm{O}$ fato de os maiores produtores utilizarem o bundling pode ser explicado por incentivos oferecidos pelas empresas a esses produtores no intuito de atraí-los.

Tendo em vista que o coeficiente da variável EPR foi não significativo, estimou-se um modelo alternativo sem essa variável, mas os resultados obtidos não foram melhores. Assim, manteve-se o modelo de equações estruturais estimado originalmente.

\section{Discussão e Conclusões}

Este artigo partiu da observação do uso de arranjos institucionais alternativos na agricultura de modo geral e no setor de fertilizantes em particular, ressaltando a crescente redução de incidência das transações no mercado spot. Procurou-se encontrar regularidades explicativas da escolha entre arranjos institucionais alternativos; assim, analisou-se a percepção dos produtores de soja com relação a diferentes arranjos institucionais para aquisição de fertilizantes, o que exigiu a realização de 200 entrevistas.

De acordo com os resultados, os produtores de soja percebem que, ao adquirir fertilizantes por meio da venda de soja para entrega futura para a mesma empresa, otimizam tempo e recursos, tendo em vista que em um mesmo momento, ocorre a transação de mais de um item. Caso o produtor não possua recursos próprios, em uma única transação ele resolve o problema do financiamento, da compra do fertilizante e da venda da soja. Essa percepção motiva o produtor a escolher esse tipo de arranjo para adquirir fertilizante. A economia nas negociações advinda da transação de um pacote de produtos reduz o que se denominou custo de negociação, que são principalmente os custos de transação ex-ante. Nesse sentido, a formatação de pacotes ou de arranjos do tipo bundling pode ser motivada por razões de eficiência, sendo a economia em custos de transação uma dessas possíveis razões. Tal visão reforça que nem todo o bundling pode ser interpretado como prática não competitiva.

A variável exposição percebida ao risco não foi significativa no modelo analisado. Com relação à aversão ao risco, os produtores mais aversos foram os que mais optaram por adquirir fertilizantes por meio 
da venda da soja para entrega futura para a mesma empresa. Com tal arranjo, no momento da aquisição do fertilizante o produtor conhece a relação de troca fertilizante/soja, ou seja, independentemente do preço da soja após a colheita, sabe quanto do seu produto será empregado para pagar o fertilizante. Parte do risco do produtor deixa de ter de ser gerenciado com o travamento do preço da soja antes do plantio, reduzindo parcialmente a incerteza do produtor.

O arranjo chamado comumente de troca, tratado neste artigo e utilizado para aquisição de fertilizante por meio da venda de soja para entrega futura, é formatado por contrato formal entre as partes. De acordo com Williamson (1996), tal arranjo é, portanto, uma forma híbrida.

De acordo com os resultados, os produtores com maior aversão ao risco utilizaram mais o arranjo troca, que é uma forma híbrida. Assim, a proposição de Williamson (1985) de que a elevada incerteza aumenta a probabilidade das estruturas de governança polares é fragilizada. No entanto, o resultado contribui mais para reforçar a colocação de Sykuta e Parcell (2002) de que a incerteza é um conceito multidimensional, sendo difícil prescrever a resposta em termos de governança sem compreender a dimensão específica pela qual a incerteza afeta a transação, o que indica a necessidade de estudos mais aprofundados sobre o tema. O arranjo abordado, embora seja uma forma híbrida, é utilizado em vez do mercado como forma de reduzir a incerteza, principalmente de preço, que está longe de representar todas as dimensões da incerteza. Além disso, o risco é utilizado como proxy para a incerteza e sabe-se que aquele não capta toda a dimensão desta. Este conceito multidimensional da incerteza é o responsável pelas controvérsias existentes na literatura com relação ao seu papel na determinação da forma de governança.

A relação encontrada entre a confiança do produtor e a utilização do pacote fertilizante/soja foi positiva. A confiança motivou os produtores de soja a utilizarem o arranjo troca, sendo que as interações repetidas entre as partes oferecem um significado central para a confiança, assim como preconizou Malhotra e Murnighan (2002). Ao escolher a utilização do pacote, o produtor aceita a relação de preço fertilizante/soja das empresas de fertilizantes, apesar de reconhecerem que estas podem deter mais informações sobre o comportamento de preços futuros da soja. No entanto, a confiança depositada nas empresas de fertilizantes baseada no 
histórico da interação das partes determina a aceitação pelo produtor dos termos de troca envolvidos no pacote. Apesar do arranjo em questão ser de curto prazo, ou seja, compreender apenas uma safra, existem evidências de que os produtores tendem a utilizá-lo em anos subseqüentes.

A relação encontrada entre a escala e a escolha do arranjo troca foi positiva. Os maiores produtores de soja, diferentemente do esperado, utilizaram mais o arranjo troca. Tal hipótese partiu da colocação de Spiller e Zelner (1997) de que a alta sofisticação do consumidor desfavorece as estratégias de bundling. Assim, os resultados contestam Spiller e Zelner (1997), embora se tenha utilizado a escala como proxy para a alta sofisticação. Essa proxy foi utilizada tendo em vista que, no caso da soja, a escala geralmente está associada ao alto nível tecnológico para plantio, manejo e colheita e aos mais diferenciados mecanismos de comercialização da safra, como, por exemplo, a negociação de contratos futuros e de opções diretamente na Bolsa de Chicago, que possui maior liquidez do que a BM\&F.

A priori, esperava-se que os maiores produtores utilizassem menos a troca, pois eles seriam mais aptos a formatarem seus próprios arranjos para proteger-se contra a incerteza e para ter acesso a crédito e a mercado. No entanto, a maior utilização do arranjo troca por parte dos grandes produtores pode ser explicada pelo lado da oferta, ou seja, as empresas de fertilizantes oferecem esse arranjo com melhores condições e incentivos para os agricultores de maior escala, o que de certa forma atua como mecanismo de incentivo. Do lado das empresas de fertilizantes, a atração de grandes produtores pode ser justificada tanto pela garantia de suprimentos com menor número de transações, quanto pelas vendas em proporções mais elevadas. Com menor número de negociações e de contratos, as empresas atingem a meta estabelecida, o que explica o seu esforço para atrair e fidelizar grandes agricultores oferecendo-lhes incentivos.

Os resultados do presente estudo devem ser analisados considerando as limitações e agenda para futuras investigações. Este artigo foi realizado com produtores de soja dos estados de Goiás e Mato Grosso que pertenciam a um cadastro particular de agricultores.

Quanto à mensuração das variáveis abordadas, acredita-se que exista espaço para melhorá-la. Embora tenham sido significativas, as 
variáveis utilizadas para custos de transação captam principalmente a parte dos custos de transação ex-ante, aqui denominado custo de negociação. Assim, seria interessante utilizar variáveis mais abrangentes que considerem os demais custos de transação ex-post. O risco não capta toda a dimensão da incerteza; assim, como se considerou apenas o risco, estimulam-se trabalhos que focalizem as diversas dimensões da incerteza para prescrever a resposta em termos de governança com base na compreensão dessas dimensões específicas pelas quais a incerteza afeta a transação.

\section{Bibliografia}

ADAMS, W.J.; YELLEN, J. (1976) Commodity bundling and the burden of monopoly. Quarterly Journal of Economics, v.90, n.3, p.475-498, 1976.

ANDERSON, J.C.; NARUS, J.A. (1990) A model of distributor firm and manufacturer firm working partnerships. Journal of Marketing, v.54, p.42-58, Jan. 1990.

CHEN, Y. (1997) Equilibrium product bundling. Journal of Business, p.85-103, 1997.

CLARO, D.P. (2003) Managing business networks and buyer-supplier relationships: How information obtained from business network affects trust, transaction specific investments, collaboration and performance in the Dutch potted plant and flower industry. Wageningen: Wageningen University and Research Centre, 2003. 196 p.

COASE, R.H. (1937) The nature of the firm. In: Williamson, O.E.; Winter, S.G. (1993) (Ed.). The nature of the firm: origins, evolution, and development. Oxford: Oxford University Press, 1993. p.18-33.

GANESAN, S. (1994) Determinants of long-term orientation in buyerseller relationship. Journal of Marketing, v. 58, p.1-19, Apr.1994.

HAIR, J.F.; ANDERSON, R.E.; TATHAM, R.L.; BLACK, W.C. (1998) Multivariate data analysis. New Jersey: Prentice Hall, 1998.

JEULAND, A. (1984) Comments on "Gaussian demand" and commodity bundling. Journal of Business, v.57, n.1, p.s231-s234, 1984. 
KNIGHT, F.H. (1921) Risk, uncertainty and profit. Chicago: University of Chicago Press, 1921. 381p.

MALHOTRA, D.; MURNIGHAN, J.K. (2002) The effects of contracts on interpersonal trust. Administrative Science Quartely, v.47, n.3, p.534-559, 2002.

NALEBUFF, B. (1999) Bundling. /Apresentado ao Seminar at the FTC, New Haven, 1999/

NOOTEBOOM, B; BERGER, H.; NOORDERHAVEN, N.G. (1997) Effects of trust and governance on relational risk. Academy of Management Journal, v.40, n.2. p.308-336, 1997.

NUNNALLY, J.C.; BERNSTEIN, I.H. (1994) Psychometric Theory. New York: McGraw-Hill, 1994.

PENNINGS, J.M.E.; LEUTHOLD, R.M. (2000) The role of farmers' behavioral attitudes and heterogeneity in futures contracts usage. American Journal of Agricultural Economy, v.82, n.4, p. 908-919, Nov. 2000.

ROCHA, M.M. (2002) Integração vertical e incerteza: um estudo empírico com a indústria petroquímica nacional. São Paulo, 2002. 159 p. Tese (Doutorado) - Faculdade de Economia, Administração e Contabilidade, Universidade de São Paulo.

SCHMALENSEE, R.; WILLIG, R.D. (1989) (Ed.) Handbook of industrial organization. Amsterdam: Elsevier Science Publishers, 1989. 658 p.

SHARMA, S.; DURVASULA, S.; DILLON, W.R. (1989) Some results on the behavior of alternate covariance structure estimation procedures in the presence of non-normal data. Journal of Marketing, v.26, p. 214-221, May 1989.

SMITH, J.B.; BARCLAY, D.W. (1997) The effects of organizational differences and trust on the effectiveness of selling partner relationships. Journal of Marketing, v.61, p.3-21, Jan. 1997.

SPILLER, P.T.; ZELNER, B.A. (1997) Product complementarities, capabilities and governance: a dynamic transaction cost perspective. Industrial and Corporate Change, v.6, n.3, p.561-595, 1997. 
SYKUTA, M.; PARCELL, J. (2002) Contract structure and design in identity preserved soybean production. CORI Working Paper, n.02-01, June 2002 .

WILLIAMSON, O.E. (1996) The mecanisms of governance. Oxford: Oxford University Press, 1996. 429 p.

WILLIAMSON, O.E. (1991) Comparative economic organization: the analysis of discrete, structural alternatives. Administrative Science Quarterly, v.36, p.269-296, June 1991.

WILLIAMSON, O.E. (1985). The economics institutions of capitalism. New York: The Free Press, 1985. 450 p. 


\section{Anexo}

\section{QUESTIONÁRIO}

\section{PESQUISA: PRODUTORES DE SOJA}

Campo: Maio de 2004

$\mathrm{N}^{\circ}$ Quest:

\section{FILTROS}

FILTRO 1: ENTREVISTAR SOMENTE PRODUTOR DE SOJA

F.1 O sr. é produtor de soja?

0. Sim (prossiga)

1. Não (encerre a entrevista)

\section{FILTRO 2: LOCALIZAÇÃO DA PROPRIEDADE DE SOJA}

F.2 Em qual estado está localizada propriedade de soja do sr. (a). Em caso de possuir mais de uma propriedade, falar da principal:

\begin{tabular}{|l|l|}
\hline 0. Goiás, OU $\quad$ (prossiga) \\
\hline 1. Mato Grosso? (prossiga) \\
\hline 2. Outros (encerre a entrevista) \\
\hline
\end{tabular}

Primeiro, eu gostaria de perguntar algumas informações gerais:

P.1 Há quantos anos o sr.(a) planta soja?

P.2 Quantos hectares de soja o sr. (a) plantou na última safra na principal propriedade? 
P.3 Ao comprar fertilizantes para a soja, como o sr.(a) fez o pagamento e qual o percentual utilizado em cada alternativa?

(Estimulada e múltipla)

0. Com recursos próprios,

1. Com recursos de financiamento bancário,

2. Com a venda de soja para entrega futura para a mesma empresa que comprei fertilizantes, (TROCA)

3. Vendi soja para entrega futura para uma empresa e depois com o recurso adquiri fertilizantes de outra empresa. OU

4. Outro (anote):

Agora, eu gostaria de perguntar sobre o que levou o sr.(a) a pagar o fertilizante da forma especificada

\section{P.4 CUSTO DE NEGOCIAÇÃO}

Vou fazer algumas afirmações e gostaria de saber se o sr.(a) concorda totalmente, apenas concorda, está indeciso, apenas discorda ou discorda totalmente. (Estimulada)

\begin{tabular}{|l|c|c|c|c|c|}
\hline \multicolumn{1}{|c|}{ FATORES } & $\begin{array}{c}\text { Discordo } \\
\text { totalmente }\end{array}$ & Discordo & Indeciso & Concordo & $\begin{array}{c}\text { Concordo } \\
\text { totalmente }\end{array}$ \\
\hline $\begin{array}{l}\text { 0. Eu tenho a } \\
\text { opção de comprar } \\
\text { fertilizantes de várias } \\
\text { marcas e empresas } \\
\text { alternativas a um raio } \\
\text { de 200 Km da minha } \\
\text { propriedade. }\end{array}$ & 1 & 2 & 3 & 4 & 5 \\
\hline $\begin{array}{l}\text { 1. Se eu comprar } \\
\text { fertilizantes e vender } \\
\text { soja para a mesma } \\
\text { empresa eu otimizo } \\
\text { meu tempo e faço } \\
\text { economia. }\end{array}$ & 1 & 2 & 3 & 4 & 5 \\
\hline $\begin{array}{l}\text { 2. É mais rápido e } \\
\text { simples, eu comprar } \\
\text { fertilizantes e vender } \\
\text { soja para uma } \\
\text { mesma empresa. }\end{array}$ & 1 & 2 & 3 & 4 & 5 \\
\hline
\end{tabular}




\section{P.5 INCERTEZA}

P.5a EXPOSIÇÃO PERCEBIDA AO RISCO

Vou fazer algumas afirmações e gostaria de saber se o sr.(a) concorda totalmente, apenas concorda, está indeciso, apenas discorda ou discorda totalmente. (Estimulada)

\begin{tabular}{|l|c|c|c|c|c|}
\hline \multicolumn{1}{|c|}{ FATORES } & $\begin{array}{c}\text { Discordo } \\
\text { totalmente }\end{array}$ & Discordo & Indeciso & Concordo & $\begin{array}{c}\text { Concordo } \\
\text { totalmente }\end{array}$ \\
\hline $\begin{array}{l}\text { 0. Eu sou capaz de } \\
\text { prever o preço da } \\
\text { soja após a colheita. }\end{array}$ & 5 & 4 & 3 & 2 & 1 \\
\hline $\begin{array}{l}\text { 1. Eu sou capaz de } \\
\text { prever a quantidade } \\
\text { a ser produzida de } \\
\text { soja ao plantar. }\end{array}$ & 5 & 4 & 3 & 2 & 1 \\
\hline $\begin{array}{l}\text { 2. O mercado da } \\
\text { soja não é arriscado. }\end{array}$ & 5 & 4 & 3 & 2 & 1 \\
\hline $\begin{array}{l}\text { 3. Eu estou exposto } \\
\text { a um risco alto } \\
\text { quando eu produzo } \\
\text { soja. (excluída da } \\
\text { análise) }\end{array}$ & 1 & 2 & 3 & 4 & 5 \\
\hline
\end{tabular}

\section{P.5b ATITUDE COM RELAÇÃO AO RISCO}

Vou fazer algumas afirmações e gostaria de saber se o sr.(a) concorda totalmente, apenas concorda, está indeciso, apenas discorda ou discorda totalmente. (Estimulada)

\begin{tabular}{|l|c|c|c|c|c|}
\hline \multicolumn{1}{|c|}{ FATORES } & $\begin{array}{c}\text { Discordo } \\
\text { totalmente }\end{array}$ & Discordo & Indeciso & Concordo & $\begin{array}{c}\text { Concordo } \\
\text { totalmente }\end{array}$ \\
\hline $\begin{array}{l}\text { 0. Eu gosto de estar } \\
\text { seguro. }\end{array}$ & 1 & 2 & 3 & 4 & 5 \\
\hline $\begin{array}{l}\text { 1. Com relação a } \\
\text { negócio, eu gosto da } \\
\text { coisa certa ao invés } \\
\text { incerta. }\end{array}$ & 1 & 2 & 3 & 4 & 5 \\
\hline $\begin{array}{l}\text { 2. Quando eu estou } \\
\text { vendendo soja eu } \\
\text { gosto de arriscar. }\end{array}$ & 5 & 4 & 3 & 2 & 1 \\
\hline
\end{tabular}


Vou fazer algumas afirmações e gostaria de saber se o sr.(a) concorda totalmente, apenas concorda, está indeciso, apenas discorda ou discorda totalmente. (Estimulada)

\begin{tabular}{|l|c|c|c|c|c|}
\hline \multicolumn{1}{|c|}{ FATORES } & $\begin{array}{c}\text { Discordo } \\
\text { totalmente }\end{array}$ & Discordo & Indeciso & Concordo & $\begin{array}{c}\text { Concordo } \\
\text { totalmente }\end{array}$ \\
\hline $\begin{array}{l}\text { 0. Eu sempre } \\
\text { compro fertilizantes } \\
\text { da mesma empresa. }\end{array}$ & 1 & 2 & 3 & 4 & 5 \\
\hline $\begin{array}{l}\text { 1. Eu compro } \\
\text { fertilizantes da } \\
\text { mesma empresa há } \\
\text { mais de dois anos. }\end{array}$ & 1 & 2 & 3 & 4 & 5 \\
\hline $\begin{array}{l}\text { 2. Eu tenho uma } \\
\text { relação de confiança } \\
\text { com a empresa } \\
\text { que eu compro } \\
\text { fertilizantes. }\end{array}$ & 1 & 2 & 3 & 4 & 5 \\
\hline
\end{tabular}

